\title{
Time delay between $\gamma$-ray lines and hard X-ray emissions during the 23 July 2002 solar flare interpreted by a trap plus precipitation model
}

\author{
C. Dauphin and N. Vilmer
}

\author{
LESIA, Observatoire de Paris, 92195 Meudon Cedex, France \\ e-mail: cyril.dauphin@obspm.fr
}

Received 14 August 2006 / Accepted 6 January 2007

\begin{abstract}
Context. The 23 July 2002 event was the first $\gamma$-ray flare observed by the Ramaty High Energy Solar Spectroscopic Imager (RHESSI). Analysis of the time profiles of the hard X-ray and gamma-ray radiations of this flare shows a time delay between hard X-ray at $150 \mathrm{keV}$ and gamma ray line emissions.

Aims. We aim to interpret this delay in terms of transport of the particles accelerated during the flare.

Methods. In this paper, we focus on the interpretation of this delay in the context of a trap plus precipitation model for energetic particles.

Results. The time profiles of hard-X-ray and prompt gamma-ray line fluxes can be reproduced given that electrons and ions are injected and partially trapped in different coronal loop systems with slightly different characteristics such as density and length, and that the energetic electron-to-ion ratio varies from peak to peak during the flare.

Conclusions. The results obtained from this analysis are discussed with respect to the constraints provided by the X-ray and gamma-ray images previously obtained, as well as with previously published analysis of the same event
\end{abstract}

Key words. Sun: flares - Sun: X-rays, gamma rays

\section{Introduction}

Hard X-ray and prompt $\gamma$-ray line radiations emitted during solar flares are a direct diagnostic of particle acceleration and transport processes. Hard X-ray emissions are produced by electronion bremsstrahlung of supra thermal electrons, and direct prompt $\gamma$-ray lines (Chupp 1984; Yoshimori 1989) are produced by the nuclear deexcitation of the ambient heavy ions after their collisions with accelerated protons and alpha particles at many $\mathrm{MeV}$ per nucleon (for review see Ramaty \& Murphy 1987). Although in most flares, non-thermal hard X-ray emissions peak at the same time (on time scales of less than a second, i.e., not considering the time of flight delays extensively studied by Aschwanden et al. 1995), analysis of particular hard X-ray bursts have shown significant delays of the peak time of hard X-ray at high energies with respect to peak times at low energies. First observations of such long delays were reported in large flares (e.g., Hoyng et al. 1976; Benz 1977; Bai \& Ramaty 1979; Vilmer et al. 1982; Bai et al. 1983). These delays can be of the order of a minute between 30 and $1800 \mathrm{keV}$. Such delays were initially interpreted in terms of a two time step acceleration mechanism (Bai \& Ramaty 1979; Bai 1982). Another interpretation (Bai \& Ramaty 1979; Emslie et al. 1979; Vilmer et al. 1982) is that these delays are produced by transport effects between the acceleration sites and the radiation sites, i.e., Coulomb energy losses of the accelerated particles in a closed coronal loop, mirroring wave interaction. These trap plus precipitation models, in which the X-ray spectrum results from the combined effects of trapping and precipitation of non-thermal electrons, have been used to explain the properties of such flares in which delays were reported (e.g., Emslie et al. 1979; Vilmer et al. 1982).
Peak time delays have also been reported in a few flares between hard X-rays and gamma ray line emissions (e.g., Hulot et al. 1989). Zweibel \& Haber (1983) were the first to compute $\gamma$-ray emissions from solar flares, taking into account the propagation of energetic ions in magnetic loops. They have shown that the mirror force traps a large fraction of the accelerated particles in the corona. As a result, the removal time of the protons from the loops could be quite long, leading to extended time profiles of $\gamma$-ray production that could be in conflict with the very impulsive observed time profiles. However, Zweibel \& Haber pointed out that if in addition to magnetic mirroring, pitch-angle scattering resulting from magnetohydrodynamic (MHD) turbulence is taken into account, then the decay-time of the gamma-ray emission from trapped-plus-precipitated protons is consistent with observed ones. Following this approach, Hua et al. (1989) computed the time dependence of the nuclear line production from ions in a magnetic loop, taking into account energy losses due to Coulomb collisions, removal by nuclear reactions, magnetic mirroring, and MHD pitch-angle scattering. However, this study did not take into account the relative timing between $\gamma$-ray and hard X-ray emissions.

Hulot and collaborators (1989) were the first ones to compute the relative time delays of $\gamma$-ray with respect to hard X-ray fluxes at different energies in a trap plus precipitation model. They show that such delays depend on both the collisional time of the protons in the loop and the precipitation rate from the loop. In particular, Hulot et al. (1992) have shown that the time delays of the hard X-ray and gamma-ray line emissions observed by ISEE-3 and GRS-SMM for the 7 June 1980 and the 27 April 1981 flares could be explained in the framework of 
this model, and parameters of the ambient medium and of the accelerated particles could be obtained. However, these studies were performed using the time profile of the 4-8 MeV range as a proxy for the time profiles of the nuclear lines and no images at X-rays or gamma-rays had been used as additional constraints to the model.

New observations at X-ray- $\gamma$-ray wavelengths are available from the Reuven Ramaty High Energy Solar Spectroscopic Imager (RHESSI; Lin et al. 2002). This spacecraft is designed to observe solar flares with high-resolution X-ray $/ \gamma$-ray imaging spectroscopy in the $3 \mathrm{keV}-17 \mathrm{MeV}$ range (Lin et al. 2002). The first gamma-ray flare observed by RHESSI was the X4.8 flare of 23 July 2002. An overview of the observations of this flare is presented in Lin et al. (2003), and more specific results on the spectroscopic and imaging analysis can be found in the special issue of The Astrophysical Journal of 1 October 2003. The analysis of the hard X-ray and gamma-ray flux time evolution exhibits a slight delay between hard X-ray emission at $150 \mathrm{keV}$ and gamma ray line emission. In this paper, we aim to analyze this delay with a trap plus precipitation model.

The paper is organized as follows. The details of the model and of the observations are recalled resp. in Sects. 2 and 3. In Sect. 4, we present the results of the interpretation of these observations in the context of the trap plus precipitation model. The last section is a discussion of the implication of these results with respect to the RHESSI observations.

\section{Trap plus precipitation models for hard X-ray and gamma-ray emissions}

This section describes the basic characteristics of the hard X-ray and $\gamma$-ray trap plus precipitation models developed by Vilmer et al. (1982) and Hulot et al. (1989) and used in this paper. As compared to the work by Hulot et al. (1989), the contribution of the alpha particles to the $\gamma$-ray line production is also included in this work. The principle of the model is the following: energetic electrons and ions are supposed to be continuously injected over a finite injection time in a coronal loop of density $n_{0}$ in which they are partially trapped. We assume that particle energy losses in the corona are only due to Coulomb collisions (see Vilmer et al. 1982; Vilmer 1987; Hulot et al. 1989). While particles trapped in the loops will produce hard X-ray and $\gamma$-ray thin target emission, electrons and protons escaping from the trap will propagate downwards to the denser chromospheric layers where they will produce hard X-ray and $\gamma$-ray line emissions in a thick target approximation. As in Hulot et al. (1989), the precipitation rate is supposed to be in the "strong diffusion limit" (see Kennel \& Petschek 1966) (the precipitation mechanism saturates). In this limit, the precipitation rate is simply related to the convergence of the magnetic field from the corona to the chromosphere and thus to the geometric size of the loss cone. The precipitation rate for a particle is finally given by (see Vilmer 1987):

$\mu_{\text {prep }}=\frac{\alpha_{0}^{2}}{2} \frac{V}{L}$

where $\alpha_{0}$ is the angle of the loss cone, $L$ is the characteristic length of the loop in $\mathrm{km}$, and $V$ is the particle velocity. The free parameters of the model are thus the precipitation rate of the trapped particles into the chromosphere, the density of the ambient medium, the spectral index and the time evolution of the

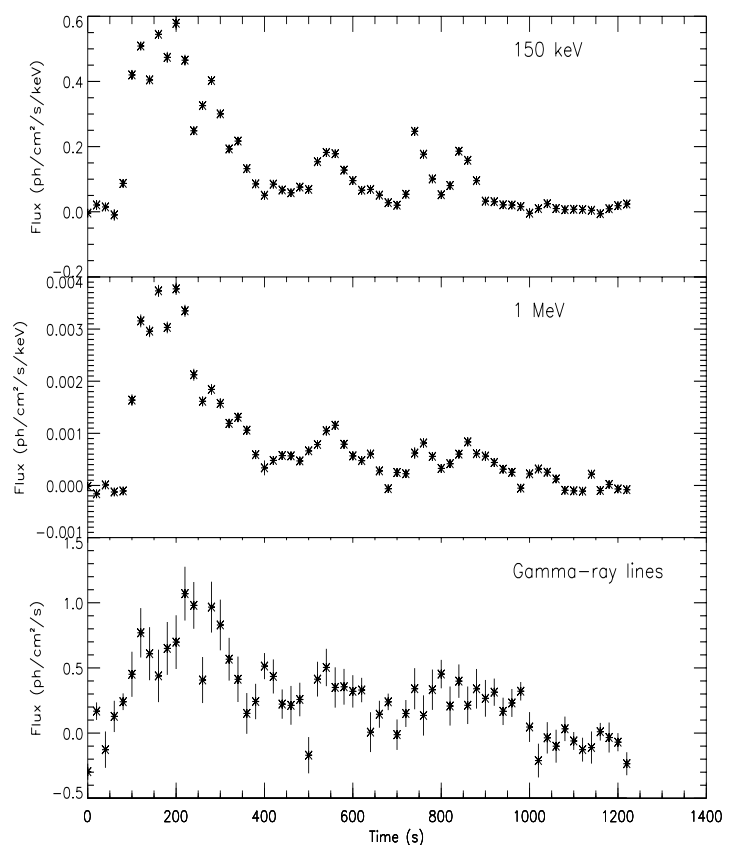

Fig. 1. Time evolution of the bremsstrahlung flux at $150 \mathrm{keV}$ and $1 \mathrm{MeV}$ and of the prompt nuclear de-excitation lines observed with a time integration of $20 \mathrm{~s}$ with RHESSI. The starting time of the figure is 00:26 UT (adapted from Share et al. 2003).

injected particles. Energetic electrons, protons, and alphas are injected in the corona at a rate given by:

$q(E, t)=q_{0} f(E) f(t)$

where $q_{0}$ is the amplitude of the injection and $f(t)$ is given by:

$f(t)=\left(t-t_{0}\right)\left(2 t_{\max }-\left(t-t_{0}\right)\right)$ for $t_{0} \leq t \leq 2 t_{\max }+t_{0}$

$f(t)=0$ elsewhere,

where $t_{\max }$ and $t_{0}$ are respectively the peak time and the starting time of the injection. $f(E)$ represents the energy spectrum of injected electrons and ions. $f(E)$ is taken as a power law in energy with a spectral parameter $\delta$ defined by:

$f(E)=E^{-\delta}$.

In the following, the electron bremsstrahlung radiation and the strong de-excitation lines at $6.129 \mathrm{MeV}$ from ${ }^{16} \mathrm{O}, 4.438 \mathrm{MeV}$ from the first excited state of ${ }^{12} \mathrm{C}$ populated directly and by the spallation reactions on ${ }^{16} \mathrm{O}, 1.779 \mathrm{MeV}$ from ${ }^{28} \mathrm{Si}, 1.634 \mathrm{MeV}$ from ${ }^{20} \mathrm{Ne}$ and $1.369 \mathrm{MeV}$ from ${ }^{24} \mathrm{Mg}$ are computed (with the photospheric target abundance for $\gamma$-ray lines). The free parameters are finally the parameter $\frac{\alpha_{0}^{2}}{L}$ linked to the precipitation rate, the density, the starting and the peak time $t_{\max }$ of the injection, the amplitude of the injection, and the spectral index of the energy spectrum of injected particles.

\section{The 23 July event}

\subsection{Temporal evolution of $X$-ray and $\gamma$-ray line emissions}

Figure 1 shows the temporal evolution of the hard X-ray emissions at $150 \mathrm{keV}$ and $1 \mathrm{MeV}$ and of the gamma-ray line time profile. These time profiles are obtained from the spectroscopic analysis presented in Share et al. (2003). The spectral analysis 

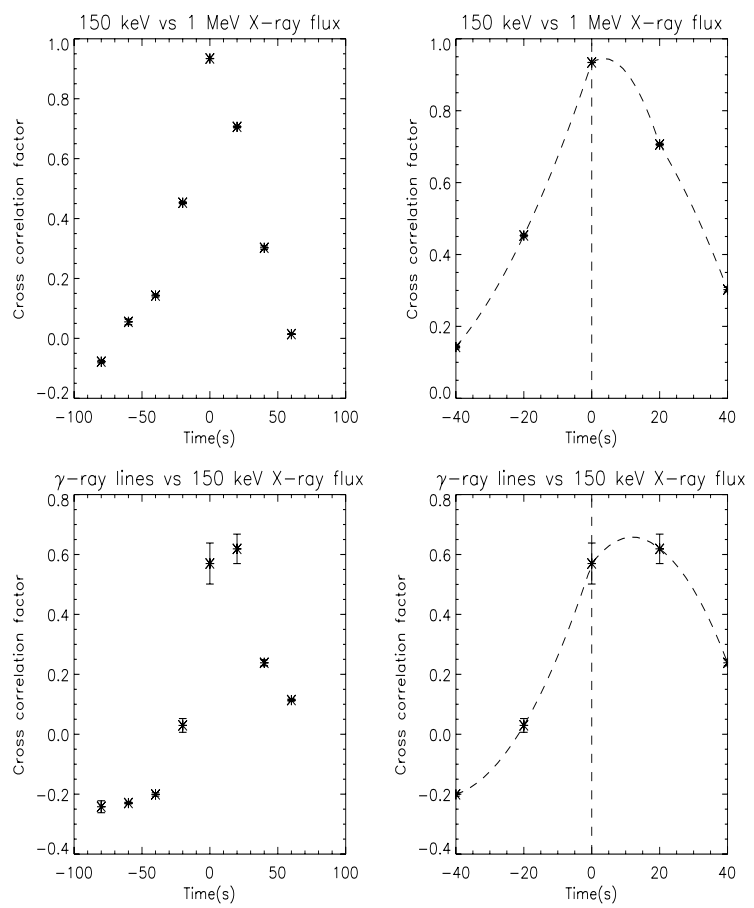

Fig. 2. Cross-correlation coefficients as a function of time lags computed from the time profiles of Fig. 1 between $150 \mathrm{keV}$ and $1 \mathrm{MeV}$ (top panels) and between $150 \mathrm{keV}$ and the gamma ray lines (bottom panels). The right panels show details for the time interval [ $-40 \mathrm{~s}, 40 \mathrm{~s}]$. The vertical dashed lines indicate a zero time lag and the dashed plot indicates the quadratic interpolation of the cross-correlation coefficients.

is performed for successive time intervals of $20 \mathrm{~s}$ and results from the fitting to observed count spectra of a model photon spectrum including a double power law for the bremsstrahlung continuum, a nuclear de-excitation line function made of 15 narrow and broad Gaussians (Smith et al. 2003), a neutron-capture line (Murphy et al. 2003), a $\alpha-{ }^{4} \mathrm{He}$ fusion line complex between $\sim 400$ and $500 \mathrm{keV}$ (Share et al. 2003), and the solar annihilation line at $511 \mathrm{keV}$ and its positronium continuum. Figure $1-$ adapted from Share et al. (2003) - shows the results of this fit as a function of time: the bremsstrahlung flux at $150 \mathrm{keV}$ and $1 \mathrm{MeV}$ and the nuclear deexcitation line flux. This figure shows that, as already observed for other flares, the hard X-ray and $\gamma$ ray time evolutions are roughly similar, indicating a common origin of the accelerated electrons and ions. These time profiles show three main parts corresponding to three successive episodes for emissions, the first one from $0 \mathrm{~s}$ to $400 \mathrm{~s}$, the second one from $450 \mathrm{~s}$ to $700 \mathrm{~s}$ and the last one from $700 \mathrm{~s}$ to $900 \mathrm{~s}$. In the following, we shall focus on the first main peak (from $0 \mathrm{~s}$ to $400 \mathrm{~s}$ ) for which a time delay is observed between the time profile at $150 \mathrm{keV}$ and the $\gamma$-ray line time profile.

Figure 2 shows the cross-correlation coefficients computed resp. between the flux at $150 \mathrm{keV}$ and $1 \mathrm{MeV}$ and between the hard X-ray flux at $150 \mathrm{keV}$ and the flux of the gamma ray line for the first main peak ( 0 to $400 \mathrm{~s}$ ). The peak value of the crosscorrelation factor is equal to one if the two time functions are of similar shape. No significant delay is observed between the peak times of the flux at $150 \mathrm{keV}$ and at $1 \mathrm{MeV}$ (see top left panel). Despite the importance of the error bars, the bottom panels show that the gamma ray peak is delayed with respect to the hard X-ray peak at $150 \mathrm{keV}$ (see bottom left panel). The dashed line in the figures on the right represents the quadratic interpolation of the cross-correlation coefficients. The delay between the

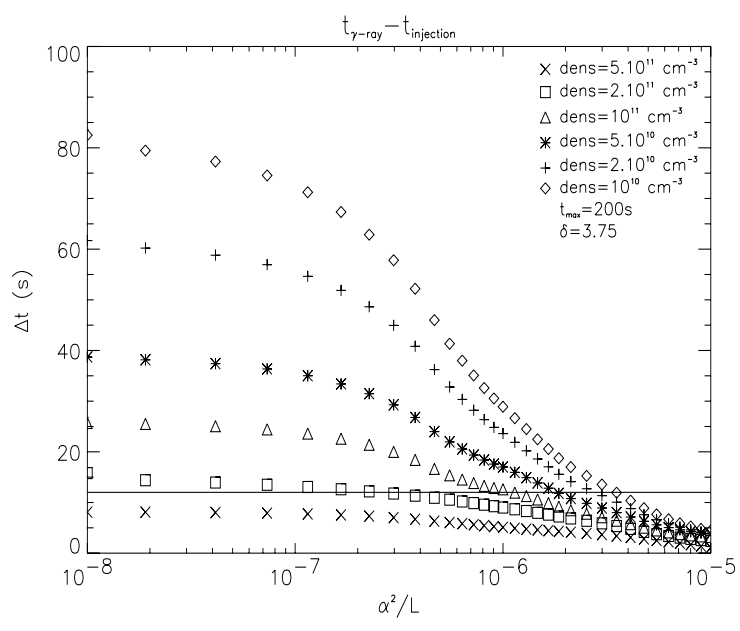

Fig. 3. Computed peak time delay $\Delta t$ between the peak time of the injection of the energetic particles and the peak time of the $\gamma$-ray line emission as expected from the trap plus precipitation models for different trap densities and as a function of $\alpha_{0}^{2} / L$. The black line represents the average observed delay of $12 \mathrm{~s}$ determined from the cross-correlation factor presented in Fig. 2.

peak time of the gamma-ray line and of the HXR flux at $150 \mathrm{keV}$ is estimated to be around $12 \mathrm{~s} \mathrm{(} \pm 6 \mathrm{~s}$ ) (see also the value in Lin et al. 2003). We shall investigate in the following whether such delays can be reproduced in the context of the trap plus precipitation model described in Sect. 2.

\subsection{First estimate of density and trap efficiency}

We make here the assumption that the time profile of the injected electrons, protons, and alpha particles follows the time profile of the hard X-ray flux at $150 \mathrm{keV}$. This assumption will be further discussed in Sect. 5. We start with the simple assumption that the time profile of the first part of the injection of energetic particles (i.e., the first part of the hard X-ray flux at $150 \mathrm{keV}$ between $0 \mathrm{~s}$ and $400 \mathrm{~s}$ ) can be simply reproduced by a parabolic injection function with a duration of $400 \mathrm{~s}\left(t_{\max }=200 \mathrm{~s}\right)$. As a first assumption, the spectral index of injected protons and alpha particles is obtained from the results reported in Lin et al. (2003). By comparing the gamma ray line fluences and assuming that the accelerated ion spectrum is a single power-law extending down to $2.5 \mathrm{Mev}_{\text {nucleon }}{ }^{-1}$, Lin et al. (2003) found an average powerlaw exponent of -3.75 for the accelerated ions. Furthermore, Smith et al. (2003) show that with this spectral index value it is also possible to reproduce the redshifts of the de-excitation lines measured. The ratio $\alpha / \mathrm{p}$ is chosen at 0.5 as in Share et al. (2003). We calculate the $\gamma$-ray flux from the five brightest de-excitation lines $\left({ }^{12} \mathrm{C},{ }^{16} \mathrm{O},{ }^{28} \mathrm{Si},{ }^{20} \mathrm{Ne}\right.$, and $\left.{ }^{24} \mathrm{Mg}\right)$.

We use the above values of the parameters to compute the delay between the peak time of the prompt $\gamma$-ray line emissions and the peak time of the injection as a function of the ratio $\alpha_{0}^{2} / L$ (proportional to the precipitation rate) and for different values of the trap density. The results for standard photospheric abundances of the target are presented in Fig. 3. They do not strongly depend on the target abundances. As expected, the peak time delay of the $\gamma$-ray lines increases with decreasing values of the ratio $\alpha_{0}^{2} / L$, i.e., with an increase of the trapping efficiency. Decreasing the trap density also leads to an increase of the delay as the collisional life time of the particles in the trap increases. Moreover, 


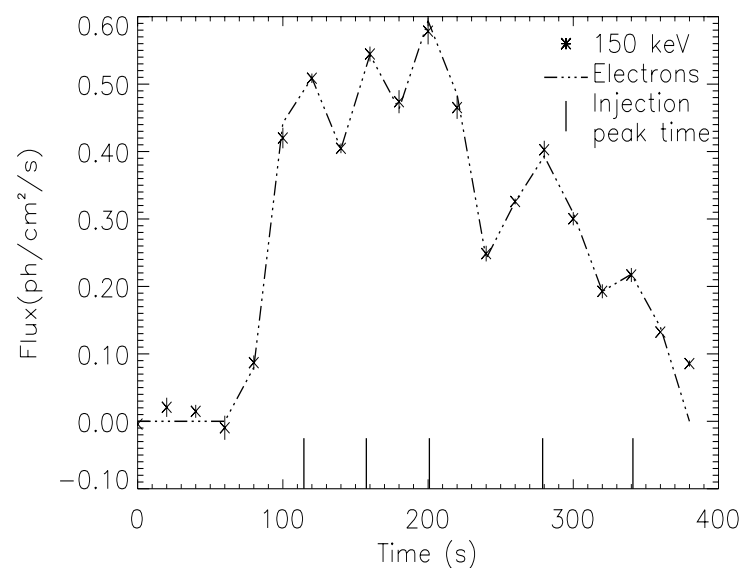

Fig. 4. Time evolution of the hard X-ray flux at $150 \mathrm{keV}$ observed with RHESSI (cross marks) and reproduction with five parabolic injections of this time evolution (dashed line). The peak time of each injection is indicated by a vertical black line. The dashed line is taken as a proxy for the time profile of the particle injection.

Fig. 3 finally shows that the observed time delay of $12 \mathrm{~s}$ can be potentially reproduced for a loop density between $2 \times 10^{11} \mathrm{~cm}^{-3}$ and $10^{10} \mathrm{~cm}^{-3}$ and a parameter $\alpha_{0}^{2} / L$ between $10^{-7} \mathrm{~km}^{-1}$ and $5 \times 10^{-5} \mathrm{~km}^{-1}$. Constraints brought by the exact reproduction of the hard X-ray and gamma-ray line temporal evolution provide a more precise determination of the value of density and trapping efficiencies of loops in which electrons and protons are injected. The following section presents a more detailed analysis of the $\mathrm{X}$-ray and $\gamma$-ray time profiles.

\section{Estimates of the trap density and efficiency from the detailed analysis of the hard X-ray and gamma-ray line time profiles}

\subsection{Detailed time profile for the injection of the energetic particles}

Compared to the previous section, we relax the assumption that the time profile of the injected electrons, protons, and alpha particles represented by the time profile at $150 \mathrm{keV}$ is a simple parabola. The first part of the time profile of the hard X-ray flux at $150 \mathrm{keV}$ is decomposed in a series of five successive parabolic injections given by Eqs. (1) and (2). Each injection is defined by three free parameters, $q_{0}, t_{0}$, and $t_{\max }$. Thus, the time profile of the injected particles is defined by fifteen parameters. The best parameters reproducing the temporal evolution of the hard X-ray flux at $150 \mathrm{keV}$ are obtained by minimizing the value of the relative $\chi^{2}$ defined by:

$\chi^{2}=\frac{1}{n-p} \sum_{i=1}^{n} \frac{\left[F_{\mathrm{inc}}\left(t_{i}, h v\right)-F_{\mathrm{cal}}\left(t_{i}, h v\right)\right]^{2}}{F_{\mathrm{err}}\left(t_{i}, h v\right)^{2}}$,

where $n$ is the number of data points (sixteen in this analysis), $p$ is the number of free parameters, $F_{\text {inc }}$ is the observed $150 \mathrm{KeV}$ flux, $F_{\text {cal }}$ is the photon flux modeled by the five injections, and $F_{\text {err }}$ is the error on the $150 \mathrm{keV}$ flux. Figure 4 shows the time evolution of the hard X-ray flux at $150 \mathrm{keV}$ and one of its best reproduction with five parabolic injections, taken in the following as a proxy for energetic particle time profile. The parameters of the different injections corresponding to a minimum of $\chi^{2}$ are reported in Table 1.
Table 1. Parameters of the five injections used to reproduce the temporal evolution of the hard X-ray flux at $150 \mathrm{keV} . q_{0_{1}}$ is the amplitude of the first injection. $q_{0_{i}}$ is the amplitude of the injection numbered $i$.

\begin{tabular}{l|ccc}
\hline \hline & $q_{0_{1, \mathrm{e}}} / q_{0_{i, \mathrm{e}}}$ & $t_{0}(\mathrm{~s})$ & $t_{\max }(\mathrm{s})$ \\
\hline Injection 1 & 1 & 77 & 37.5 \\
Injection 2 & 0.34 & 137 & 20.5 \\
Injection 3 & 1.23 & 156 & 44.5 \\
Injection 4 & 1.95 & 233 & 45.5 \\
Injection 5 & 1.68 & 310 & 31.5 \\
\hline
\end{tabular}

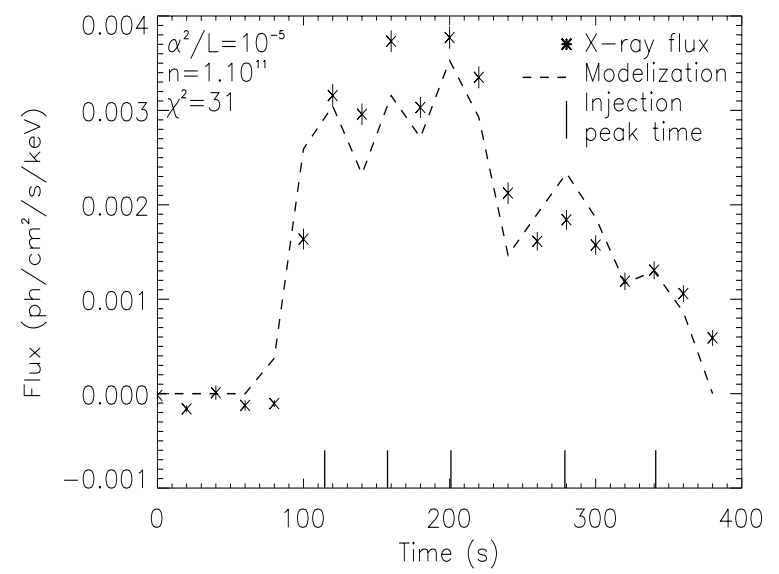

Fig. 5. Time evolution of the hard X-ray flux at $1 \mathrm{MeV}$ observed with RHESSI (cross marks) and obtained from the trap plus precipitation (dashed line) with $n=10^{11} \mathrm{~cm}^{-3}, \alpha_{0}^{2} / L=10^{-5} \mathrm{~km}^{-1}$, and $\delta=-4$ for each injection. The peak time of each injection is indicated with a vertical black line.

\subsubsection{Temporal evolution of the $1 \mathrm{MeV}$ bremsstrahlung flux}

In this section, we predict the time evolution of the bremsstrahlung flux at $1 \mathrm{MeV}$ given the five electron injections of Table 1. It is assumed that the production of energetic electrons at all energies follow the same injection time profile with the same values for the ratio between each $q_{0}$. The analysis is done in two different steps.

1. In a first step, the electron spectral index is kept constant for the five injections. Assuming that the continuum emission up to $1 \mathrm{MeV}$ is produced by thick-target bremsstrahlung, the spectral analysis presented in Holman et al. (2003) shows that during the time interval studied, the spectral index of the injected electrons is around $\delta=4$ above around $100 \mathrm{keV}$. We simply assume here that the spectral index of the injected electrons keeps this value up to several MeVs. Figure 5 shows the time evolution of the flux at $1 \mathrm{MeV}$ obtained from the trap plus precipitation with $n=10^{11} \mathrm{~cm}^{-3}, \alpha_{0}^{2} / L=$ $10^{-5} \mathrm{~km}^{-1}$, and $\delta=4$ for each injection. This is one of the best fits obtained by varying the density between $n=$ $10^{9} \mathrm{~cm}^{-3}$ and $n=10^{12} \mathrm{~cm}^{-3}$, and $\alpha_{0}^{2} / L$ between $\alpha_{0}^{2} / L=$ $10^{-7} \mathrm{~km}^{-1}$ and $\alpha_{0}^{2} / L=10^{-5} \mathrm{~km}^{-1}$. The computed values present several discrepancies compared to measured values, notably for the second and the fourth injections. Similar discrepancies are found for other values of the parameters. A slightly different spectral index for the injected electrons for these injections could explain these differences. 
2. In a second step, we thus relax the assumption of a constant spectral index for each injection. For each injection, the injected spectral index is determined so as to reproduce the amplitude of the $1 \mathrm{MeV}$ flux at the peak time. To minimize the effect of the transport, we determine these values in the case of a large density $\left(n=1 \times 10^{11} \mathrm{~cm}^{-3}\right)$ and of a large escape rate $\left(\alpha_{0}^{2} / L=10^{-5} \mathrm{~km}^{-1}\right)$. The values of the spectral in$\operatorname{dex} \delta$ found for the different injections using this method are reported in the first column of Table 2 . The subtle variation of $\delta$ from injection to injection is consistent with the result of the spectral analysis by Holman et al. (2003), who have found a slightly larger electron spectral index at the time of the fourth injection (see Fig. 3 of their paper).

The values of the spectral indices for electron injections reported in Table 2 are used to compute the time profile of the bremsstrahlung flux at $1 \mathrm{MeV}$ for different values of the density $\left(n=5 \times 10^{9} \mathrm{~cm}^{-3}, n=1 \times 10^{10} \mathrm{~cm}^{-3}, n=5 \times 10^{10} \mathrm{~cm}^{-3}\right.$, and $n=1 \times 10^{10} \mathrm{~cm}^{-3}$ ) and several values of $\alpha_{0}^{2} / L$ between $10^{-7} \mathrm{~km}^{-1}$ and $10^{-5} \mathrm{~km}^{-1}$. For each couple of trap density and efficiency values, the amplitude of the injections are determined by minimizing the relative $\chi^{2}$.

Figure 6 presents the time evolution of the observed flux at $1 \mathrm{MeV}$, of the computed one for $n=1 \times 10^{10} \mathrm{~cm}^{-3}$ and $n=1 \times$ $10^{11} \mathrm{~cm}^{-3}$, and for three different values of $\alpha_{0}^{2} / L 7.5 \times 10^{-6} \mathrm{~km}^{-1}$, $5 \times 10^{-6} \mathrm{~km}^{-1}$, and $2.5 \times 10^{-6} \mathrm{~km}^{-1}$. Several comments can be made:

1. The time profile at $1 \mathrm{MeV}$ is reproduced for a high precipitation rate. The different figures indeed show that the peak of the $1 \mathrm{MeV}$ flux corresponding to the second injection is not well reproduced for a parameter $\alpha_{0}^{2} / L$ lower than $5 \times$ $10^{-6} \mathrm{~km}^{-1}$.

2. Such a high precipitation rate implies in fact that most of the electrons precipitate without being trapped on long time scales and that the bremsstrahlung emission is mostly produced in the same way as in a "thick target". As a consequence, the results are not strongly dependent on the values of the density.

3. The best reproduction of the time evolution of the $1 \mathrm{MeV}$ flux is obtained for $\alpha_{0}^{2} / L$ around $5 \times 10^{-6} \mathrm{~km}^{-1}$ and a density comprised between $1 \times 10^{10} \mathrm{~cm}^{-3}$ and $1 \times 10^{11} \mathrm{~cm}^{-3}$. The second injection is lost in the case of a smaller escape rate (larger trapping rate).

In the second column of Tables 2 and 3, we give the values of the number of electrons injected above $150 \mathrm{keV}$ for $\alpha_{0}^{2} / L=5 \times$ $10^{-6} \mathrm{~km}^{-1}$ and, respectively, $n=1 \times 10^{10} \mathrm{~cm}^{-3}$ and $n=1 \times$ $10^{11} \mathrm{~cm}^{-3}$. The number of electrons is calculated by integrating Eq. (2) on the time duration of each injection. This analysis leads to a total number of $\sim 4.2 \times 10^{36}$ electrons injected above $150 \mathrm{keV}$ during the first part of the event. This number will be discussed in Sect. 5.

\subsubsection{Temporal evolution of the gamma ray lines}

In this section, we examine whether the time evolution of the gamma-ray line flux can be reproduced with the five injections given in Table 1, taking into account transport effects. As recalled in Sect. 4.2, the spectral index chosen for accelerated protons and alpha particles is 3.75 in agreement with values deduced for the same event from different analysis (e.g., Lin et al. 2003). The ratio $\alpha / \mathrm{p}$ is taken as 0.5 . The time profiles of the gamma ray lines are computed for different values of the trap density
Table 2. Parameters of the five injections used to reproduce the temporal evolution of the hard X-ray flux at $1 \mathrm{MeV}$ for $\alpha_{0}^{2} / L=5 \times 10^{-6} \mathrm{~km}^{-1}$ and $n=1 \times 10^{10} \mathrm{~cm}^{-3} . \delta$ is the spectral index value.

\begin{tabular}{l|cc}
\hline \hline & $\delta$ & Nbr electrons \\
\hline Injection 1 & 4.01 & $1.1 \times 10^{36}$ \\
Injection 2 & 4 & $5.5 \times 10^{35}$ \\
Injection 3 & 4.01 & $1.5 \times 10^{36}$ \\
Injection 4 & 4.05 & $8.1 \times 10^{35}$ \\
Injection 5 & 4.02 & $3.6 \times 10^{35}$ \\
\hline
\end{tabular}

$n=5 \times 10^{9} \mathrm{~cm}^{-3}, n=1 \times 10^{10} \mathrm{~cm}^{-3}, n=5 \times 10^{10} \mathrm{~cm}^{-3}$, and $n=1 \times 10^{11} \mathrm{~cm}^{-3}$, and several values of the parameter $\alpha_{0}^{2} / L$ between $10^{-7} \mathrm{~km}^{-1}$ and $10^{-5} \mathrm{~km}^{-1}$. In these conditions, the additional free parameters left to reproduce the gamma-ray line time profiles are the relative amplitudes of the different injections.

In a first step, the relative amplitude of each injection was kept as given in Table 1, i.e., the relative number of injected electrons and ions is kept constant from injection to injection. For each couple of trap density and parameter $\alpha_{0}^{2} / L$, the amplitude $q_{0_{1}}$ is determined so as to reduce $\chi^{2}$. Figure 7 shows the time evolution of the observed and computed nuclear de-excitation lines for several couples of values. From these figures, it is obvious that keeping the same relative amplitudes of the injections as for the electron injections does not allow us to reproduce the observed time profile of the gamma-ray line flux. In particular, while the second injection produces a $\gamma$-ray line flux that is too strong, the fourth injection does not produce one that is strong enough.

In a second step, the assumption that the relative number of electrons and ions is kept constant from injection to injection is relaxed. For each couple of trap densities and paramater $\alpha_{0}^{2} / L$, the relative amplitudes of each injection presented in Table 4 are then determined so as to minimize the relative $\chi^{2}$. Figure 8 displays the comparison of the time evolution of the observed and computed gamma-ray lines obtained in such conditions. A better reproduction of the time evolution of the gamma-ray lines is clearly obtained in these cases. The minimum relative $\chi^{2}$ and the better reproduction of the $\gamma$-ray flux is obtained for $n=1 \times$ $10^{10} \mathrm{~cm}^{-3}$ and $\alpha^{2} / L=10^{-6} \mathrm{~km}^{-1}$. Table 4 summarizes the values of the amplitudes $q_{0_{i}}$ and the numbers of protons and alpha particles injected above $1 \mathrm{MeV}$ for each injection in this case. This analysis leads to a total number of $\sim 3.5 \times 10^{37}$ protons and alpha particles injected above $1 \mathrm{MeV}$ during the first part of the event. This number will be discussed in Sect. 5 .

The dependence of the results on the spectral index of injected ions was also analyzed and an analysis similar to the one presented above was achieved for $\delta=3$ and $\delta=4.5$. Table 5 summarizes the results similarly obtained for $\delta=3$ and $\delta=4.5$. The number of injected ions above $1 \mathrm{MeV}$ increases with a steeper spectrum, since the $\gamma$-ray line emission is essentially produced by ions above a few $\mathrm{MeV}$ and is not strongly dependent on the shape of the spectrum.

For each injection, Table 6 summarizes the relative number of injected electrons above $150 \mathrm{keV}$ when compared to the total number of particles (electrons above $150 \mathrm{keV}$ plus protons and alphas above $1 \mathrm{MeV} / \mathrm{nuc}$ ) deduced from Tables 2 and 4, i.e., for the cases $n=1 \times 10^{10} \mathrm{~cm}^{-3}, \alpha^{2} / L=5 \times 10^{-6}$ for electrons and $n=1 \times 10^{10} \mathrm{~cm}^{-3}, \alpha^{2} / L=10^{-6}$ for ions. It can be noticed that injections 2 and 5 are characterized by a larger number of electrons as compared to ions. Such a variability of electron to ion production has been observed previously in flares based on 

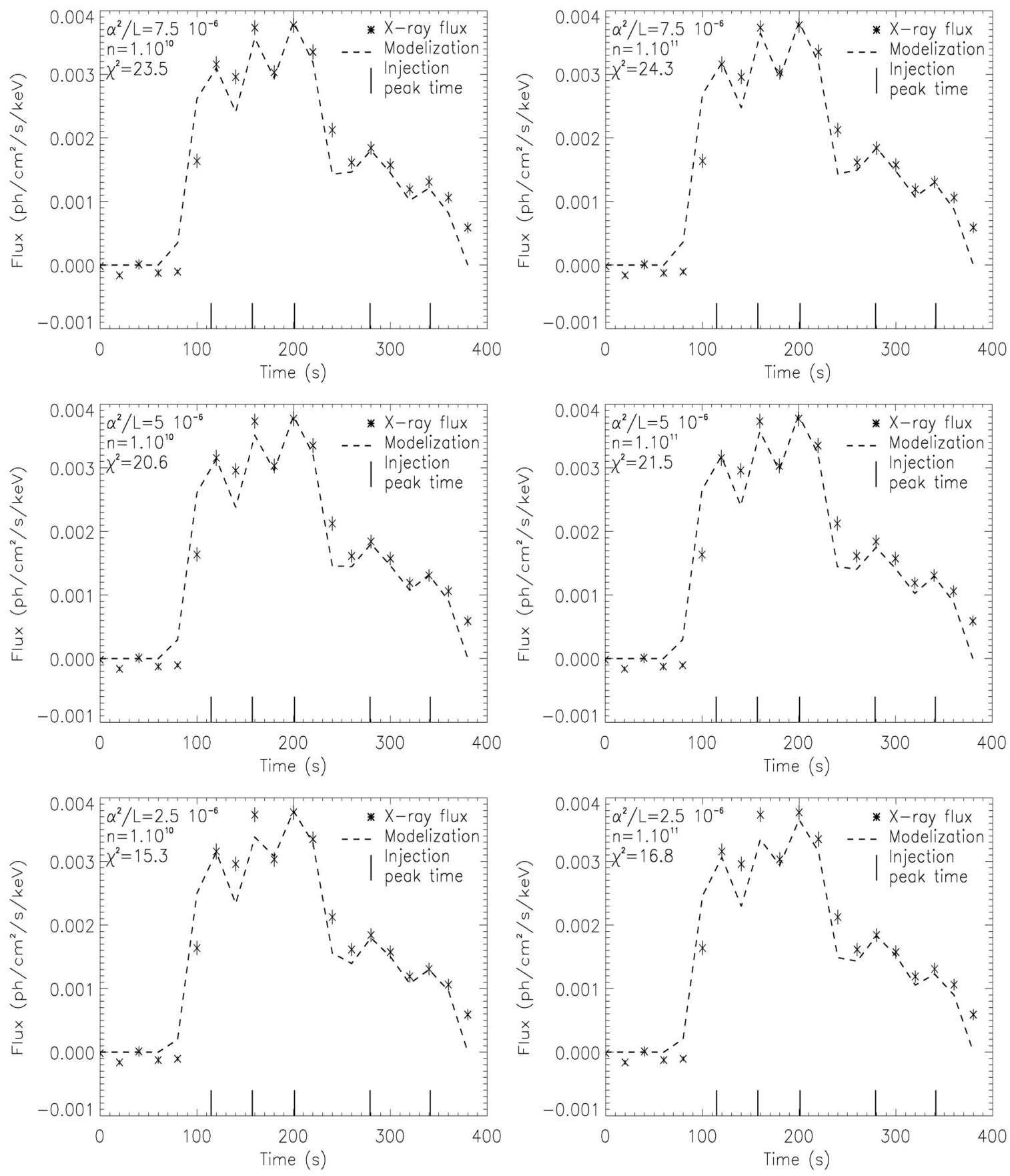

Fig. 6. Time evolution of the bremsstrahlung flux at $1 \mathrm{MeV}$ observed with RHESSI (cross marks) and obtained from the trap plus precipitation model (dashed line) for different values of the density and of the paramater $\alpha_{0}^{2} / L$ indicated in each panel. The black thick lines indicate the time of each injection. The ratio between the amplitude of each injection is given in Table 1.

spectral analysis (e.g., Chupp et al. 1993). A similar result is recovered here from the time delay analysis. This will be further discussed in Sect. 5.

The relative production of $\gamma$-ray line emission from the trapi.e., the coronal loops - and the footpoints are finally computed given the parameters found for the trap plus precipitation model. In the case with $\delta=3.75, n=1 \times 10^{10} \mathrm{~cm}^{-3}$, and $\alpha^{2} / L=$ $10^{-6} \mathrm{~km} \mathrm{~s}^{-1}$, it is found that $28 \%$ of the total $\gamma$-ray flux is produced in the loop, while $72 \%$ is produced at the footpoints. This result is similar for each injection. As a conclusion, even if partial trapping effects of energetic ions may explain the time delays of gamma-ray lines with respect to X-ray emissions, this analysis
Table 3. Same as Table 2, but for $\alpha_{0}^{2} / L=5 \times 10^{-6} \mathrm{~km}^{-1}$ and $n=1 \times$ $10^{11} \mathrm{~cm}^{-3}$.

\begin{tabular}{l|cc}
\hline \hline & $\delta$ & Nbr electrons \\
\hline Injection 1 & 4.01 & $1.1 \times 10^{36}$ \\
Injection 2 & 4 & $5.4 \times 10^{35}$ \\
Injection 3 & 4.01 & $1.4 \times 10^{36}$ \\
Injection 4 & 4.05 & $7.8 \times 10^{35}$ \\
Injection 5 & 4.02 & $3.7 \times 10^{35}$ \\
\hline
\end{tabular}

shows that most of the $\gamma$-ray flux still comes from the footpoints and not from coronal sources. 

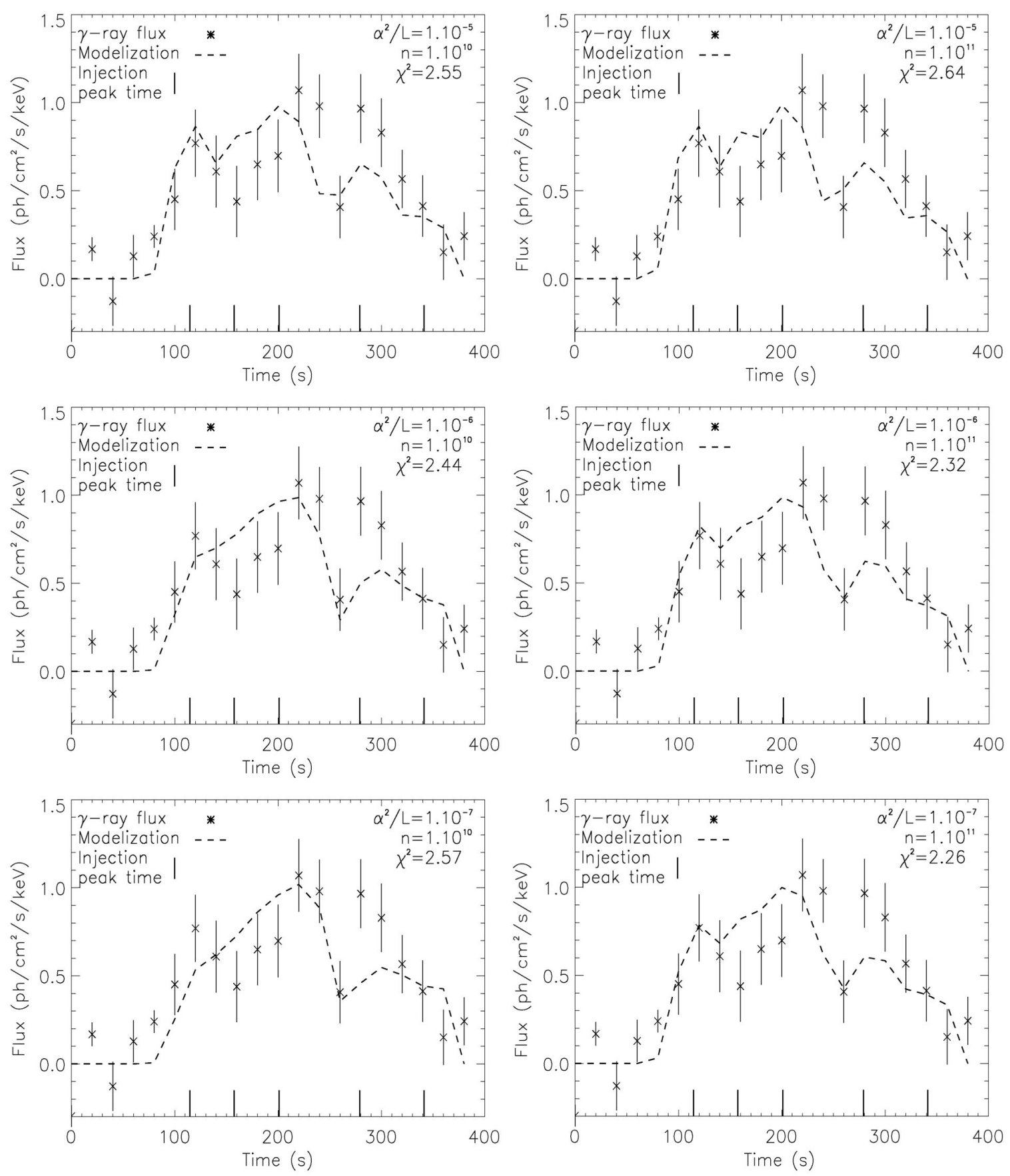

Fig. 7. Time evolution of the observed and computed gamma ray flux for $n=10^{10} \mathrm{~cm}^{-3}$ and $n=10^{11} \mathrm{~cm}^{-3}$, and for three values of $\alpha^{2} / L 10^{-5} \mathrm{~km}{ }^{-1}$, $10^{-6} \mathrm{~km}^{-1}$, and $10^{-7} \mathrm{~km}^{-1}$. For each plot, the dashed line corresponds to the computed gamma ray flux and the cross marks correspond to the observations. The vertical black lines indicate the time of each injection. The relative amplitude for each injection is the same as in Table 1 .

\section{Discussion and conclusion}

The 23 July 2002 flare was the first $\gamma$-ray flare observed with RHESSI. We present in this paper an interpretation of the relative temporal evolution of the hard X-ray flux at $150 \mathrm{keV}$ and $1 \mathrm{MeV}$ and of the prompt nuclear de-excitation lines in the context of a trap plus precipitation model. A clear time delay around $12 \mathrm{~s}$ is indeed observed between the hard X-ray flux at $150 \mathrm{keV}$ and the $\gamma$-ray flux, while no significant delay is observed between the hard X-ray flux at $1 \mathrm{MeV}$ and at $150 \mathrm{keV}$.

Assuming that the time profile of the injected electrons, protons, and alpha particles follows the time profile of the hard
$\mathrm{X}$-ray flux at $150 \mathrm{keV}$, we have shown as a first step that such delays between hard X-ray emission at $150 \mathrm{keV}$ and prompt gamma-ray lines can be reproduced by a trap plus precipitation model for a density between $2 \times 10^{11} \mathrm{~cm}^{-3}$ and $10^{10} \mathrm{~cm}^{-3}$ and a value of $\alpha^{2} / L$ between $10^{-7} \mathrm{~km}^{-1}$ and $5 \times 10^{-5} \mathrm{~km}^{-1}$. In a second step, we have considered that the time profile of the hard $\mathrm{X}$-ray flux at $150 \mathrm{keV}$ and thus of the particle injection is decomposed in a series of five successive parabolic injections. The time profile of both the $1 \mathrm{MeV}$ bremsstrahlung flux and of the prompt gamma-ray line flux can then be reproduced in the context of the 

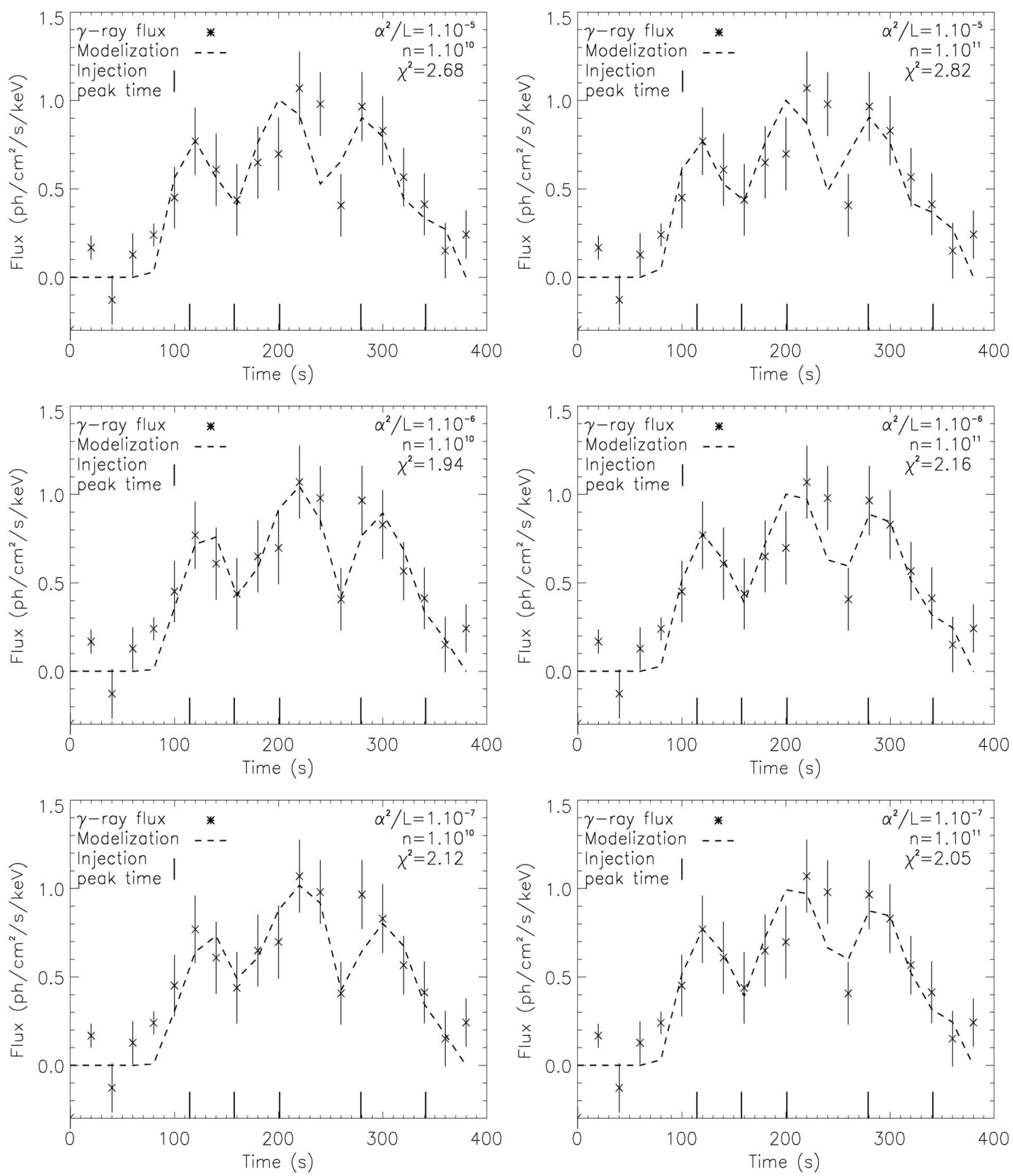

Fig. 8. Same as Fig. 7, but the relative values of the amplitudes of the injections are determined so as to minimize $\chi^{2}$.

Table 4. Relative amplitudes of the five injections and number of protons and $\alpha$ particles above $1 \mathrm{MeV}$ necessary to reproduce the temporal evolution of the $\gamma$-ray flux for $n=1 \times 10^{10} \mathrm{~cm}^{-3}$ and $\alpha^{2} / L=10^{-6} \mathrm{~km}^{-1}$ and $\delta=3.5$.

\begin{tabular}{l|cc}
\hline \hline & $q_{0_{1, \mathrm{p}}} / q_{0_{\mathrm{i}, \mathrm{p}}}$ & Nbr $p+\alpha$ \\
\hline Injection 1 & 1 & $9.3 \times 10^{36}$ \\
Injection 2 & 12.2 & $9.5 \times 10^{34}$ \\
Injection 3 & 1.17 & $1.3 \times 10^{37}$ \\
Injection 4 & 1.37 & $1.2 \times 10^{37}$ \\
Injection 5 & 19.3 & $3.1 \times 10^{35}$ \\
\hline
\end{tabular}

Table 5. Same as Table 4, but for $\delta=-3$ and $\delta=-4.5$.

\begin{tabular}{l|cc|cc}
\hline \hline & \multicolumn{2}{|c|}{$\delta=3$} & \multicolumn{2}{c}{$\delta=4.5$} \\
& $q_{0_{1, \mathrm{p}}} / q_{0_{\mathrm{i}, \mathrm{p}}}$ & Nbr $p+\alpha$ & $q_{0_{1, \mathrm{p}}} / q_{0_{\mathrm{i}, \mathrm{p}}}$ & $\mathrm{Nbr} p+\alpha$ \\
\hline Injection 1 & 1 & $2.8 \times 10^{36}$ & 1 & $2.0 \times 10^{37}$ \\
Injection 2 & 58.6 & $7.9 \times 10^{33}$ & 3.5 & $1.0 \times 10^{36}$ \\
Injection 3 & 1.15 & $4.0 \times 10^{36}$ & 1.15 & $2.9 \times 10^{37}$ \\
Injection 4 & 1.34 & $3.7 \times 10^{36}$ & 1.38 & $2.6 \times 10^{37}$ \\
Injection 5 & 219 & $8.1 \times 10^{33}$ & 6.2 & $2.0 \times 10^{36}$ \\
\hline
\end{tabular}


Table 6. Percentage of electrons above $150 \mathrm{keV}$ compared to the total number of particles (electrons above $150 \mathrm{keV}$ and protons and alphas above $1 \mathrm{MeV} /$ nuc for each injection).

\begin{tabular}{l|c}
\hline \hline & Elec $\geq 150 \mathrm{keV} /$ nbr particles \\
\hline Injection 1 & $11 \%$ \\
Injection 2 & $85 \%$ \\
Injection 3 & $11 \%$ \\
Injection 4 & $7 \%$ \\
Injection 5 & $54 \%$ \\
\hline
\end{tabular}

trap plus precipitation model given the following assumptions and leading to the following results:

1. We use the time profile of the hard X-ray flux at $150 \mathrm{keV}$ as the particle injection time profile. Although weaker trapping effects are expected for the energetic electrons producing the hard X-ray flux at $150 \mathrm{keV}$, time delays can still be observed in this energy range between the injection and the emission time profiles. To confirm our assumption, we verify that the $150 \mathrm{keV}$ hard X-ray time profile computed with the density and precipitation rate deduced above is not delayed compared to the injection.

2. We use a ratio $\alpha / p$ equal to 0.5 (Share et al. 2003). We find similar delays for a ratio equal to 0.1 or to 0.9 .

3. The spectral index for the injected electrons and ions is mainly determined from the previously published spectral analysis of the hard X-ray and $\gamma$-ray fluxes. A minor variation of the spectral index of the injected electrons is, however, found from one injection to the other to better reproduce the time profile of the $1 \mathrm{MeV}$ flux. This small variation is in fact consistent with the result of the spectral analysis by Holman et al. (2003), who found a slightly larger electron spectral index at the time of the fourth injection. The hard $\mathrm{X}$-ray time profile at $1 \mathrm{MeV}$ is then correctly reproduced if electrons are injected in a loop (or loop system) with a density comprised between $n=1 \times 10^{10} \mathrm{~cm}^{-3}$ and $n=1 \times$ $10^{11} \mathrm{~cm}^{-3}$ and a value of $\alpha_{0}^{2} / L$ around $5 \times 10^{-6} \mathrm{~km}^{-1}$. In these conditions, hard X-ray emissions are almost produced in a thick target approximation leading to negligible time delays between the hard X-ray flux at $150 \mathrm{keV}$ and at $1 \mathrm{MeV}$. Electrons are thus found to propagate in a loop with a density consistent with the thermal plasma density of $6 \times 10^{10} \mathrm{~cm}^{-3}$ of the coronal X-ray source observed at $12-25 \mathrm{keV}$ in the rise phase of the flare (Lin et al. 2003). The estimation of the plasma density was done from the emission measure determined from GOES measurements at the end of the rise phase (Holman et al. 2003) combined to the size of the $12-25 \mathrm{keV}$ hard X-ray source observed with RHESSI just before the flare also assumed to be the size of the thermal source (Lin et al. 2003).

4. The study furthermore shows that if the global time delay between the $\gamma$-ray and hard X-ray flux can be explained in the context of the simple trap-plus precipitation model, the detailed time profile of the $\gamma$-ray line evolution can only be reproduced if the electron to ion production varies from injection to injection. A strong decrease of the relative number of ions above $1 \mathrm{MeV}$ when compared to the number of electrons above $150 \mathrm{keV}$ is thus found for the second and fifth injections. Such variations between the relative number of interacting electrons and ions during the course of a flare have been already reported by Chupp et al. (1993). Such a result implies either different proton and electron acceleration regions, or an acceleration mechanism with a relative efficiency for accelerating protons or electrons varying with time. In these conditions, a good reproduction of the time evolution of the prompt gamma-ray lines is obtained with a value of $\alpha^{2} / L$ of $10^{-6} \mathrm{~km}^{-1}$ and a density of $1 \times 10^{10} \mathrm{~cm}^{-3}$.

Thus - compared to electrons - ions seem to be injected in a system of coronal loops of lower density and with a slightly smaller value of $\alpha^{2} / L$ leading to a slightly more efficient trapping. If we consider a similar value for the loss cone for the two coronal loops, we find that electrons and ions propagate in coronal loops of different lengths with a ratio $L_{\mathrm{e}} / L_{\mathrm{p}, \alpha}=0.2$. This result must be compared with what can be deduced from the hard X-ray and gamma-ray line images of the flare combined with the coronal loop images provided by the TRACE satellite (Transition Region And Coronal Explorer). Postflare (90 min) images indeed show a network of long magnetic structures, with one end coinciding well with the centroid of the $2.223 \mathrm{MeV}$ emission (see Fig. 4 of Lin et al. 2003). Emslie et al. (2004) suggested furthermore that if the postflare magnetic structures are indicative of the geometry of the ion acceleration region, then the associated length is $70^{\prime \prime} \approx 5 \times 10^{9} \mathrm{~cm}$. The hard X-ray sources and the coronal source in the impulsive phase are inside these large-scale loops (Lin et al. 2003), likely on an inner arcade of loop. From the footpoint separations, the hard X-rays are on the other hand produced in much smaller loops with lengths on the order of $20^{\prime \prime}$ or $1.5 \times 10^{9} \mathrm{~cm}$. The ratio of the length of the coronal loops in which electrons and protons propagate is thus estimated from these considerations to be $L_{\mathrm{e}} / L_{\mathrm{p}}=1.5 / 5$ (Emslie et al. 2004), which is consistent with the ratio found from our analysis.

Further comments on the interpretation of Emslie et al. (2004) on the location of electron and ion acceleration sites can be made from the present analysis. Their interpretation is based on the assumption that electrons and ions are accelerated by waves generated by MHD turbulence (Miller \& Roberts 1995) in loops. For proton acceleration to dominate, this model requires that the escape time from the loop be greater than the acceleration time and leads to the following criterium:

$15 n^{1 / 2} L>\frac{B^{2}}{Q^{2 / 3}}$,

where $n$ is the density normalized to $10^{10} \mathrm{~cm}^{-3}, L$ the loop length normalized to $10^{9} \mathrm{~cm}, B$ the magnetic field normalized to $10^{2} \mathrm{G}$, and $Q$ the rate of density energy injected normalized to $10^{2} \mathrm{erg} \mathrm{cm}^{-3} \mathrm{~s}^{-1}$. If our present analysis seems to confirm the difference of length between the loops in which electrons and ions, the density of the coronal loops in which protons are injected may be weaker than that of the electrons.

The number of energetic electrons, protons, and alpha particles produced in the first part of the flare can be deduced from our analysis. The total number of electrons above $150 \mathrm{keV}$ injected during the first part of the event is found to be of the order of $\sim 4.2 \times 10^{36}$, corresponding to an energy content in accelerated electrons above $150 \mathrm{keV}$ around $1.5 \times 10^{30} \mathrm{erg}$. These values are in good agreement with the results found from the spectral analysis of Holman et al. (2003). Assuming a thick target production of hard X-ray emissions, they found a rate of energy deposition by the non thermal electrons above $20 \mathrm{keV}$ around $2 \times 10^{28} \mathrm{erg} \mathrm{s}^{-1}$ in the impulsive phase, which corresponds to $\sim 6 \times 10^{30}$ erg deposited by the non thermal electrons during the first part of the event. The total energy in protons and alpha particles injected above $2.5 \mathrm{MeV} /$ nuc found from our model is around $\sim 2 \times 10^{31} \mathrm{erg}$. The analysis of Lin et al. (2003) leads to a similar energy content in all accelerated ions above $2.5 \mathrm{MeV}$ of $\sim 10^{31} \mathrm{erg}$. 
The relative production of $\gamma$-ray line emission from the trapi.e., the coronal loops - and the footpoints derived from our analysis is also computed. It is found that given the parameters of the coronal loop in which ions are injected, most of the $\gamma$-ray flux $(>70 \%)$ still comes from the footpoints and that coronal emission represents less than $30 \%$ of the flux. Thus, if images of the prompt $\gamma$-ray lines were available with a similar resolution as the images of the $2.2 \mathrm{MeV}$ line, relatively compact sources should be observed.

In conclusion, we underline the interest of the RHESSI imaging capability to further constrain the interpretation of time delays in the context of trap plus precipitation models. Future studies will be to extend this kind of analysis to other gamma ray events observed with RHESSI for which gamma-ray emissions not only comes from footpoints, but also from the corona (Krucker and Hurford, RHESSI science nugget).

\footnotetext{
Acknowledgements. Cyril Dauphin and Nicole Vilmer acknowledge support from the Centre National d'Etudes Spatiales on the RHESSI project. The authors would like to thank $\mathrm{H}$. Hudson and G. Hurford for useful discussions and $\mathrm{G}$. Share for providing the X-ray and gamma-ray time profiles resulting from the spectroscopic analysis.
}

\section{References}

Aschwanden, M. J., Schwartz, R. A., \& Alt, D. M. 1995, ApJ, 447, 923 Bai, T. 1982, ApJ, 259, 341

Bai, T., \& Ramaty, R. 1979, ApJ, 227, 1072

Bai, T., Dennis, B. R., Kiplinger, A. L., et al. 1983, Sol. Phys., 86, 409 Benz, A. O. 1977, ApJ, 211, 270

Chupp, E. L. 1984, ARA\&A, 22, 359

Chupp, E. L., Trottet, G., Marschhauser, H., et al. 1993, A\&A, 275, 602 Emslie, A. G., McCaig, M. G., \& Brown, J. C. 1979, Sol. Phys., 63, 175 Emslie, A. G., Miller, J. A., \& Brown, J. C. 2004, ApJ, 602, 69 Holman, G. D., Sui, L., Schwartz, R. A., et al. 2003, ApJ, 595, 97 Hoyng, P., Brown, J. C., \& Van Beek, H. F. 1976, Sol. Phys., 48, 197 Hua, X. M., Ramaty, R., \& Lingenfelter, R. E. 1989, ApJ, 341, 516 Hulot, E., Vilmer, N., \& Trottet, G. 1989, A\&A, 213, 383

Hulot, E., Vilmer, N., Chupp, E. L., et al. 1992, A\&A, 256, 273 Kennel, C. F., \& Petscheck, H. E. 1966, JGR, 71, 1

Lin, R. P., \& the RHESSI team. 2002, Sol. Phys., 210, 3

Lin, R. P., Krucker, S., Hurford, G. J., et al. 2003, ApJ, 595, 69 Miller, A. J., \& Roberts, A. D. 1995, ApJ, 452, 912

Murphy, R. J., Share, G. H., Hua, H.-M., et al. 2003, ApJ, 595, 93 Ramaty, R., \& Murphy, R. J. 1987, Space Sci. Rev., 45, 213 Share, G. H., Murphy, R. J., Skibo, J. G., et al. 2003, ApJ, 595, 85 Smith, D. M., Share, G. H., Murphy, R. J., et al. 2003, ApJ, 595, 81 Vilmer, N. 1987, Sol. Phys., 111, 207

Vilmer, N., Trottet, G., \& Kane, S. R. 1982, A\&A, 108, 306

Yoshimori, M. 1989, Space Sci. Rev., 51, 85

Zweibel, E. G., \& Haber, D. 1983, ApJ, 264, 648 